\title{
A Theoretical Investigation of Polymer-Nanoparticles as Miscibility Improvers in All-Polymer Nanocomposites
}

\author{
Alaitz Ruiz de Luzuriaga ${ }^{1, a}$, Hans Grande ${ }^{2, b}$ and Jose A. Pomposo ${ }^{3, c}$ \\ 1,2,3 New Materials Department, CIDETEC, Paseo Miramón 196, \\ Donostia E-20009, Spain \\ aaruiz@cidetec.es, bhgrande@cidetec.es, \\ cjpomposo@cidetec.es (corresponding author)
}

received paper 12.12.2008, received revised paper, 05.01.2008accepted date 06.012008

Keywords: nanoparticles; nanocomposites; phase diagram; miscibility.

\begin{abstract}
The miscibility behaviour of polymer-nanoparticle / linear-polymer blends (all-polymer nanocomposites) has been investigated using an incompressible mean-field theoretical model that accounts for combinatorial, temperature-dependent exchange interaction energy and nanoparticle-driven effects. The theory is employed to predict the phase diagram of poly(styrene)-nanoparticle (PS-np) / linear-poly(vinyl methyl ether) (PVME) nanocomposites from room temperature to $675 \mathrm{~K}$. Complete miscibility is predicted for PS-nanoparticles with radius $<6 \mathrm{~nm}$ blended with PVME (molecular weight $62500 \mathrm{~g} / \mathrm{mol}$, nanoparticle volume fraction $20 \%$ ). The effect of PVME molecular weight and blend composition on the miscibility diagram is also addressed. When compared to the well-known experimental phase diagram of linear-PS / PVME blends displaying lower critical solution temperature (LCST) behaviour, the miscibility improving effect of sub-10 nm PS-nanoparticles is clearly highlighted. In terms of the model, this favourable nanoscale effect arises mainly from the reduced stretching induced by the sub-10 nm nanoparticles and the increased exothermic contacts when compared to nanoparticles with sizes $>10 \mathrm{~nm}$.
\end{abstract}

\section{Introduction}

The interest in the synthesis of nanoparticles is growing exponentially due to their new properties arising form nanoscale effects and their potential use in different applications covering from Nanomedicine to Nanoelectronics [1]. Although the synthesis of metal and metal oxide nanoparticles in the sub-10 nm size range is well established, 
the synthesis of polymer-nanoparticles with diameter $<10 \mathrm{~nm}$ is just an emerging field [2-4]. The lack of versatile synthetic routes to cross-linked individual polymernanoparticles has certainly delayed the development of all-polymer nanocomposites, i.e. polymer-nanoparticle / linear-polymer blends. Currently, intramolecular cross-linking of individual polymer chains at very dilute concentration is the main synthetic route to collapsed polymer-nanoparticles in the 3-15 nm size range [2]. For all-polymer nanocomposites new and interesting nanoscale effects are expected. A good example is the non-Einstein rheological behaviour reported for athermal poly(styrene)-nanoparticle (PS-np) / linear-poly(styrene) (PS) nanocomposites in the melt state [3, 4]. Fine-tuning of the polymer-nanoparticle size becomes crucial to observe such unexpected behaviour. Another example is the miscibility observed in blends of poly(ethylene)-nanoparticle (PE-np) and PS [1], even when binary linear-polyethylene (PE) / PS blends are well known to be immiscible.

Recently, we have developed an entropic model for predicting the phase behaviour of athermal all-polymer nanocomposites [5a]. A comparison of the predictive theory and reported experimental data has been performed by using the poly(styrene)-nanoparticle (PS-np) / linear-poly(styrene) (PS) pair as a model system. The excellent agreement observed indicated that dilution of contact nanoparticle-nanoparticle interactions (instead of a nanoscale surface enthalpy gain) plays a key role in explaining the miscibility behaviour of cross-linked nanoparticles dispersed in a chemically identical linear polymer matrix [5a].

In this paper, we extend our recent theory to treat weakly interacting all-polymer nanocomposites. The new incompressible mean-field theoretical model introduced accounts for combinatorial, temperature-dependent exchange interaction energy and nanoparticle-driven effects. The theory is employed to predict the phase diagram of poly(styrene)-nanoparticle (PS-np) / linear-poly(vinyl methyl ether) (PVME) nanocomposites. Theoretical predictions indicate that sub-10 nm PS-nanoparticles should be actually miscibility improvers in PS-np / PVME nanocomposites.

\section{Theory}

Let us consider a weakly interacting all-polymer nanocomposite, composed of spherical polymer-nanoparticles (component 1 ) of radius $R_{p}$, nanoparticle volume $v_{1}$ and linear-polymer chains of degree of polymerization $N_{2}$, monomer volume $v_{2}$, and radius of gyration $R_{g B} \approx 2 r_{B} N_{B}^{1 / 2} / \sqrt{6}$, being $r_{2}$ the radius of a repeat unit. 
By accounting for the changes in free energy arising from combinatorial $\left(\Delta g_{m i x}^{c o}\right)$, nanoparticle-driven $\left(\Delta g_{m i x}^{n p}\right)$ and temperature-dependent exchange interaction energy $\left(\Delta g_{m i x}^{e x}\right)$ effects, an expression for the free energy of mixing per unit volume is obtained such as

$$
\Delta g_{m i x}=\Delta g_{m i x}^{c o}+\Delta g_{m i x}^{n p}+\Delta g_{m i x}^{e x}
$$

where

$$
\begin{aligned}
& \Delta g_{\text {mix }}^{c o}=k T\left[\frac{\phi_{1}}{v_{1}} \ln \phi_{1}+\frac{\phi_{2}}{N_{2} v_{2}} \ln \phi_{2}\right] \\
& \Delta g_{m i x}^{n p}=k T\left[\frac{\phi_{1}}{v_{1}}\left(\frac{4 \phi_{1}-3 \phi_{1}^{2}}{\left(1-\phi_{1}\right)^{2}}\right)+\frac{3}{2} \times \frac{\phi_{1} \phi_{2}}{N_{2} v_{2}}\left(\frac{R_{p}}{R_{g B}}\right)^{2}\right]
\end{aligned}
$$

and

$$
\Delta g_{m i x}^{e x}=\left(\frac{r_{2}}{R_{p}}\right)\left[\frac{k T}{v_{2}}\left(\chi_{S}+\frac{\chi_{H}}{T}\right)\right] \phi_{1} \phi_{2}
$$

In eq. $1 \mathrm{a}-\mathrm{c}, k$ is the Boltzmann constant, $T$ the absolute temperature, $\phi_{i}$ is the volume fraction of component $i$ and $\chi_{S}$ and $\chi_{H}$ are the entropic and enthalpic components of the blend interaction parameter $\left(\chi=\chi_{S}+\chi_{H} / T\right)$, respectively.

Eq. 1a-b has been reported previously by treating athermal all-polymer nanocomposites [5a]. Eq. 1a provides the contribution to the total free energy of mixing due to the combinatorial entropy of mixing whereas eq. $1 \mathrm{~b}$ gives the contribution to the free energy of mixing due to nanoparticle-driven effects. Eq. $1 \mathrm{~b}$ takes into account the dilution of hard sphere-like nanoparticle-nanoparticle interactions upon mixing (first rhs term) and the stretching of the linear-polymer chains due to the presence of the nanoparticles (second rhs term). Referring to this latter term, a Ginburg-type expansion term is adopted here to account for the fact that the polymer-nanoparticles cause stretching of the polymer chains in their vicinity [6]. This term is insensitive to the chemical nature of the linear-chains and it is only dependent on chain length and nanoparticle size.

Eq. 1c accounts for the temperature- and nanoparticle size-dependent interaction effects in the weakly interacting all-polymer nanocomposite. As in Ginzburg incompressible model [6], a prefactor $\left(r_{2} / R_{p}\right)$ is introduced in eq. $1 \mathrm{c}$ since the number of surface contacts with monomers 2 for each nanoparticle becomes smaller as one 
increases the nanoparticle radius. This ratio tends toward unity inasmuch $R_{p} \rightarrow r_{1}$, as it must.

Equation 1 is presumably valid only for $\phi_{1}<0.494$ (the theoretical maximum nanoparticle packaging volume fraction without freezing) [5b] and for $R_{p}>\sim 5 r_{2}\left(r_{2}\right.$ is typically around $0.3 \mathrm{~nm}$ ). When $R_{p} \approx r_{2}$ the nanoparticle-driven terms should be equal to zero since then the nanoparticles would be equivalent to monomers 1 . In the intermediate range $\left(r_{2}<R_{p}<5 r_{2}\right)$ an interpolating factor should be introduced as discussed previously by Ginzburg [6].

It is well known that for a binary blend to be thermodynamically stable against phase splitting, the following conditions must be fulfilled: $\Delta g_{m i x}<0$ and $\Delta g_{m i x}^{(2)} \equiv\left(\frac{\partial^{2} \Delta g_{m i x}}{\partial \phi_{1}^{2}}\right)>$ 0 . The condition $\Delta g_{\text {mix }}^{(2)}=0$ just determines the spinodal miscibility boundary in the phase diagram. In terms of the model, it is given by

$$
\Delta g_{m i x}^{(2)}=\Delta g_{m i x}^{c o(2)}+\Delta g_{m i x}^{n p(2)}+\Delta g_{m i x}^{e x(2)}=0
$$

where

$$
\begin{aligned}
\Delta g_{\text {mix }}^{c o(2)} & =k T\left[\frac{1}{v_{1} \phi_{1}}+\frac{1}{N_{2} v_{2} \phi_{2}}\right] \\
\Delta g_{\text {mix }}^{n p(2)} & =k T\left[\frac{2}{v_{1}}\left(\frac{4-\phi_{1}}{\left(1-\phi_{1}\right)^{4}}\right)-\frac{9}{2 v_{2}}\left(\frac{R_{p, 0}}{r_{2} N_{2}}\right)^{2}\right]
\end{aligned}
$$

and

$$
\Delta g_{m i x}^{e x(2)}=-2\left(\frac{r_{2}}{R_{p}}\right)\left[\frac{k T}{v_{2}}\left(\chi_{S}+\frac{\chi_{H}}{T}\right)\right]
$$

\section{Results and discussion}

Binary blends of PS and PVME phase separate upon heating through a lower critical solution temperature (LCST) phase behaviour $[7,8]$. As an example, for PS $\left(M_{n} \sim 500\right.$ $000 \mathrm{~g} / \mathrm{mol}) / \operatorname{PVME}\left(M_{n} \sim 50000 \mathrm{~g} / \mathrm{mol}\right)$ blends at a volume fraction of PS of $20 \%$, the LCST is experimentally found at $\sim 375 \mathrm{~K}$. Modelling of the LCST of binary PS / PVME blends has been performed by means of different theoretical approaches such as latticefluid equation-of-state [9] or lattice cluster theories [10]. Alternatively, a simpler mean field binary interaction model with temperature-dependent interaction parameters was 
used to estimate the interaction energy between PS and PVME [11, 12]. Hence, modelling of the LCST phase boundary of PVME / poly(styrene-co-methyl methacrylate) blends provided a reliable temperature-dependent interaction parameter for the PS / PVME pair: $\chi \approx 0.015-15 / T$. As an example, by using this temperature-dependent interaction parameter, the predicted LCST for the above PS / PVME blend is $370 \mathrm{~K}$.

In this work we are interested to investigate, from a theoretical point of view, the effect on the PS / PVME phase diagram of changing one of the components from a linear-polymer (e.g. linear-PS) to a polymer-nanoparticle (e.g. PS-nanoparticle). The predicted spinodal miscibility boundary for PS-nanoparticle / PVME nanocomposites as a function of nanoparticle radius is illustrated in Fig. 1.

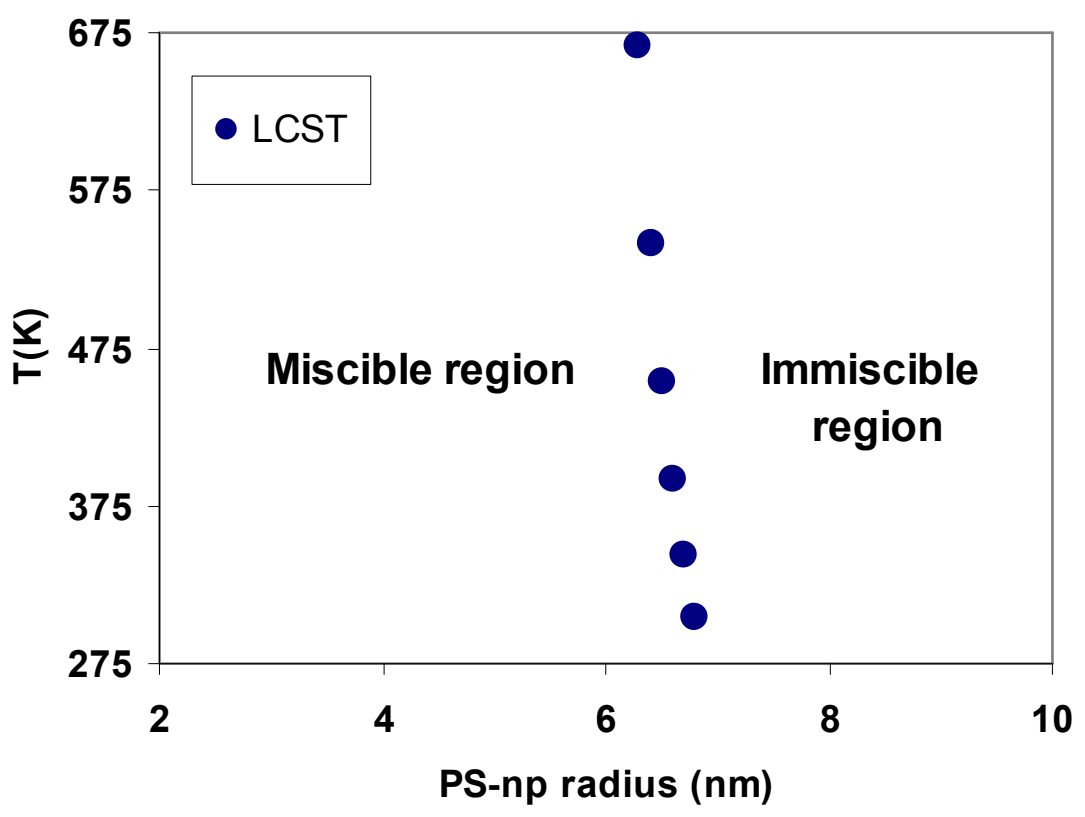

Fig. 1. Predicted phase diagram for PS-np / PVME $\left(M_{n}=62500 \mathrm{~g} / \mathrm{mol}\right)$ nanocomposites as a function of nanoparticle size as calculated from eq. 2 by using $\chi \approx 0.015-15 / T, \phi_{1}=0.2, N_{2}=1079, v_{2}=46.36 \mathrm{~cm}^{3} / \mathrm{mol}$ and $r_{2}$ $=0.26 \mathrm{~nm}$.

Interestingly, complete miscibility across the 275 - 675 temperature range is calculated for PS-nanoparticles with radius $<6 \mathrm{~nm}$. Conversely, for PS-nanoparticles with radius $>7 \mathrm{~nm}$ complete phase separation is expected. For PS-nanoparticles of 6.5 $\mathrm{nm}$ in radius, the presence of a LCST at $454 \mathrm{~K}$ is predicted. 
Fig. 2 illustrates the evolution of $\Delta g_{\text {mix }}^{c o(2)}, \Delta g_{m i x}^{n p(2)}, \Delta g_{m i x}^{e x(2)}$ and $\Delta g_{\text {mix }}^{(2)}$ (see eq. 2) as a function of temperature for PS-np / PVME nanocomposites with PS-nanoparticles of 6.5 $\mathrm{nm}$ in radius. As can be seen in Fig. 2, $\Delta g_{m i x}^{c o(2)}$ is always positive (e.g. favourable to mixing) and increases linearly with temperature, as expected (see eq. 2a). Conversely, $\Delta g_{m i x}^{n p(2)}$ is always negative following also a linear trend with $\mathrm{T}$ (see eq. $2 \mathrm{~b}$ ).

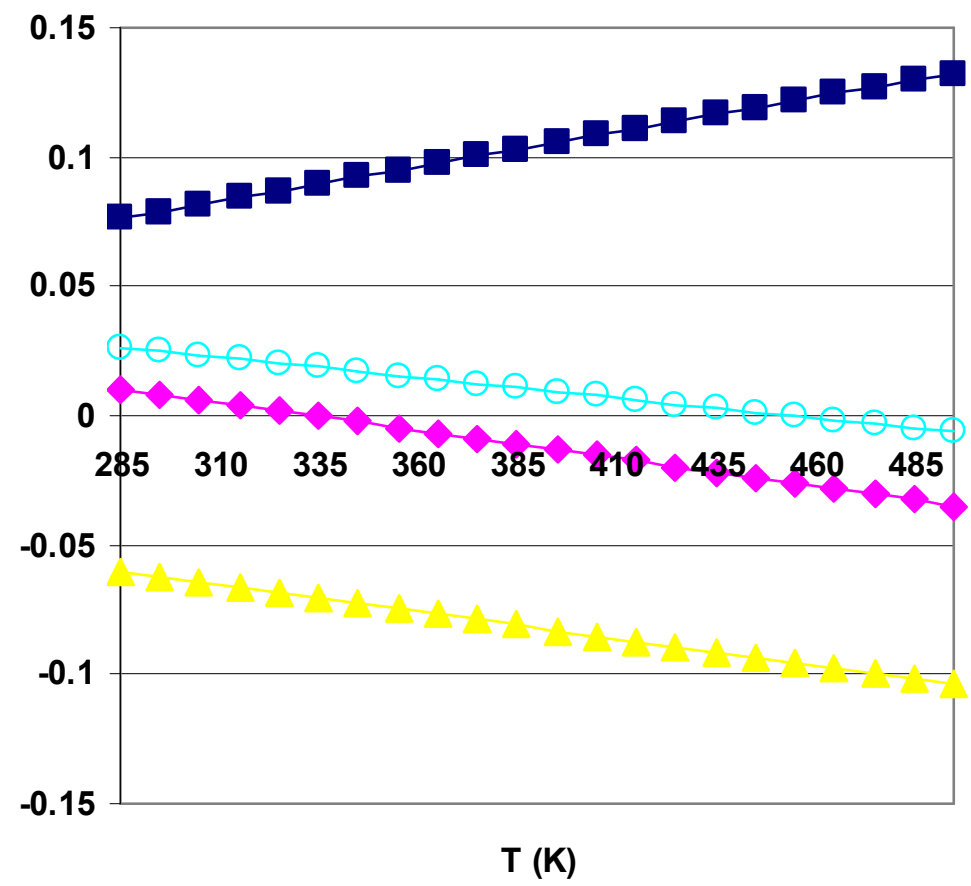

Fig. 2. Calculated values of $\Delta g_{\text {mix }}^{c o(2)}\left(\mathrm{J} / \mathrm{cm}^{3}\right)$ (solid squares), $\Delta g_{m i x}^{n p(2)}\left(\mathrm{J} / \mathrm{cm}^{3}\right)$ (solid triangles), $\Delta g_{\text {mix }}^{e x(2)}\left(\mathrm{J} / \mathrm{cm}^{3}\right) \quad$ (solid diamons) and $\Delta g_{m i x}^{(2)}=\Delta g_{m i x}^{c o(2)}+\Delta g_{m i x}^{n p(2)}+\Delta g_{m i x}^{e x(2)}\left(\mathrm{J} / \mathrm{cm}^{3}\right)$ (open circles) as a function of temperature for PS-np / PVME $\left(M_{n}=62500 \mathrm{~g} / \mathrm{mo}\right)$ nanocomposites with PS-nanoparticles of $6.5 \mathrm{~nm}$ in radius at $\phi_{1}=0.2$ (see eq. 2).

According to the model, for PS-nanoparticles of $6.5 \mathrm{~nm}$ in radius the unfavourable stretching effects arising from the nanoparticle size overcome the favourable dilution of hard sphere-like nanoparticle-nanoparticle interactions upon mixing resulting in $\Delta g_{\text {mix }}^{n p(2)}<$ 0 . On the other hand, $\Delta g_{m i x}^{e x(2)}$ changes from positive to negative values upon increasing temperature, since at high temperatures $\chi_{S}>\left|\chi_{H} / T\right|$. As a result, $\Delta g_{\text {mix }}^{(2)}$ changes from positive to negative values at $454 \mathrm{~K}$ (LCST behaviour). 
For PS-nanoparticles of radius $<6 \mathrm{~nm}$, the favourable dilution of hard sphere-like nanoparticle-nanoparticle interactions upon mixing clearly overcomes the small unfavourable stretching effects arising from the reduced nanoparticle size resulting in $\Delta g_{\text {mix }}^{n p(2)}>0$. The physical meaning is that sub-10 nm nanoparticles do not disturb significantly the conformation of the linear polymer chains to induce a strong entropy penalty $[5 \mathrm{a}, 6]$. In addition, the increased exothermic contacts when compared to nanoparticles with radii $>6 \mathrm{~nm}$ (accounted for by the $\left(r_{2} / R_{p}\right)$ factor in eq. $2 \mathrm{c}$ [6]) make the $\Delta g_{\text {mix }}^{e x(2)}$ term to take more positive values. The opposite is true for PS-nanoparticles of radius $>6 \mathrm{~nm}$.

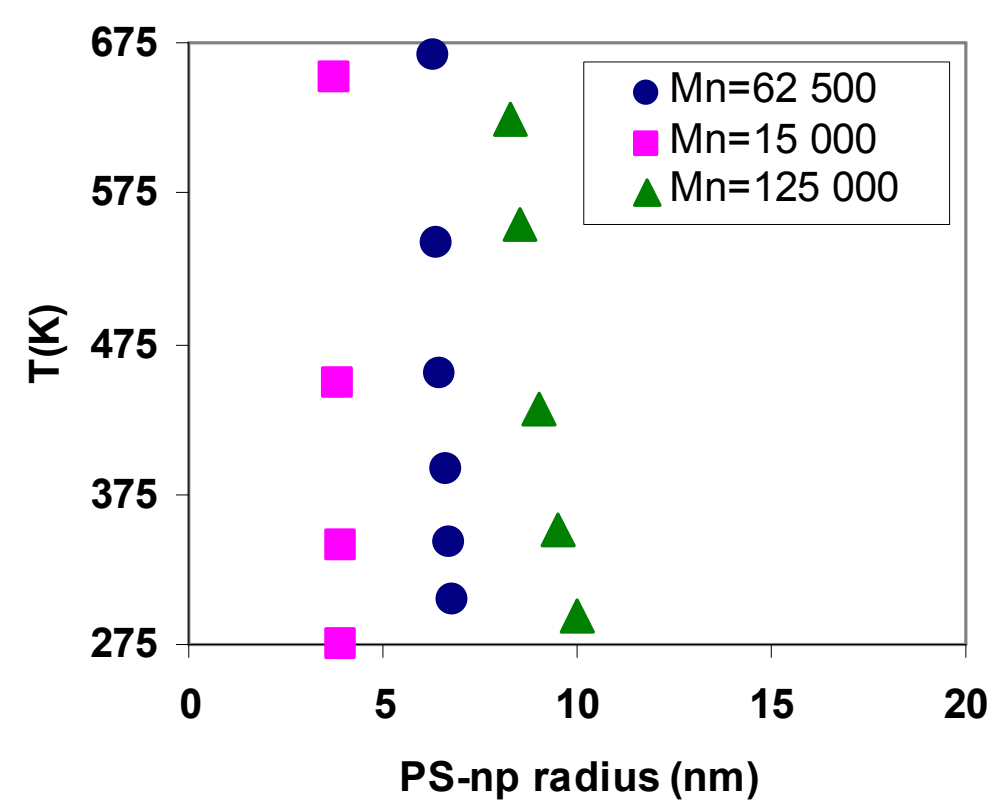

Fig. 3. Influence of PVME molecular weight on the calculated phase diagram for PS-np / PVME nanocomposites $\left(\phi_{1}=0.2\right): M_{n}=15000 \mathrm{~g} / \mathrm{mol}$ (solid squares), $M_{n}=62500 \mathrm{~g} / \mathrm{mol}$ (solid circles) and $M_{n}=125000 \mathrm{~g} / \mathrm{mol}$ (solid triangles).

We have also investigated the effect of PVME molecular weight and blend composition on the phase diagram of PS-np / PVME nanocomposites.

As can be seen in Figure 3, for PVME with $M_{n}=15000 \mathrm{~g} / \mathrm{mol}$ complete miscibility in the $275-675$ temperature range is obtained for PS-np with radii $<3.8 \mathrm{~nm}$. Upon increasing the PVME molecular weight to $125000 \mathrm{~g} / \mathrm{mol}$, miscibility is predicted for PS$\mathrm{np}$ with radii $<8.3 \mathrm{~nm}$. According to the model, the entropy penalty for nanoparticle 
inclusion into the linear-polymer matrix (second rhs term in eq. $1 b$ and $2 b$ ) decreases for larger linear-polymer chains $\left(N_{B} \propto R_{g B}^{2}\right)$ so larger nanoparticles can be accommodated.

We found no significant differences in critical nanoparticle radius for immiscibility as function of composition for PS-np / PVME $\left(M_{n}=62500 \mathrm{~g} / \mathrm{mol}\right)$ nanocomposites. As an example, complete miscibility at $298 \mathrm{~K}$ is predicted for nanocomposites with $R_{p}<6.7$ $\mathrm{nm}, R_{p}<6.8 \mathrm{~nm}$ and $R_{p}<7.4 \mathrm{~nm}$ at $\phi_{1}=0.1, \phi_{1}=0.2$ and $\phi_{1}=0.3$, respectively.

\section{Conclusions}

We have performed a theoretical study of the effect of polymer-nanoparticles on the phase behaviour of weakly interacting all-polymer nanocomposites. An incompressible mean-field theoretical model that accounts for combinatorial, temperature-dependent exchange interaction energy and nanoparticle-driven effects has been introduced and employed to predict the phase behaviour of PS-np / PVME nanocomposites.

Complete miscibility is expected for PS-nanoparticles with radius $<6 \mathrm{~nm}$ blended with PVME $\left(M_{n}=62500 \mathrm{~g} / \mathrm{mol}\right)$ at $\phi_{1}=0.2$. In the nanocomposites, the critical nanoparticle radius for immiscibility was found to increase with PVME molecular weight. As an example, complete miscibility was predicted for PS-np with $R_{p}<3.8 \mathrm{~nm}$ and PVME with $M_{n}=15000 \mathrm{~g} / \mathrm{mol}$. For PVME with $M_{n}=125000 \mathrm{~g} / \mathrm{mol}$, miscibility is expected for PS-np with $R_{p}<8.3 \mathrm{~nm}$. Conversely, differences in blend composition (at low nanoparticle volume fractions for which the model is valid) showed not a pronounced effect on the critical nanoparticle radius for immiscibility.

When compared to the well-known experimental phase diagram of linear-PS / PVME blends displaying lower critical solution temperature (LCST) behaviour, the miscibility improving effect of sub-10 nm PS-nanoparticles was clearly highlighted. In terms of the model, this favourable nanoscale effect arises mainly from the reduced stretching induced by the sub-10 $\mathrm{nm}$ nanoparticles and the increased exothermic contacts when compared to nanoparticles with $R_{p}>10 \mathrm{~nm}$.

We are currently extending the present study to cover other polymer-nanoparticle / linear-polymer systems using well-established temperature-dependent binary interaction parameters. A check of the theoretical predictions and experimental data will be certainly valuable. 


\section{Acknowledgments}

Financial support by MEC (Grant No. CSD2006-53), Basque Government and Diputación de Gipuzkoa through CIC NANOGUNE-CONSOLIDER, IKERTU Fellowship Program and NANOTRON Project is gratefully acknowledged.

\section{References and notes}

[1] A. C. Balazs, T. Emrick, T. P. Russell, Nanoparticle polymer composites: where two small worlds meet, Science 314 (2006) 1107-1110.

[2] E. Harth, B. v. Horn, V. Y. Lee, D. S. Germack, C. P. Gonzales, R. D. Miller, C. J. Hawker, A facile approach to architecturally defined nanoparticles via intramolecular chain collapse, J. Am. Chem. Soc. 124 (2002) 8653-8660.

[3] M. E. Mackay, T. T. Dao, A. Tuteja, D. L. Jo, B. v. Horn, H-C. Kim, C. J. Hawker, Nanoscale effects leading to non-Einstein-like decrease in viscosity, Nat. Mater. 2 (2003) 762-766.

[4] A. Tuteja, M. E. Mackay, C. J. Hawker, B. v. Horn, Effect of ideal, organic nanoparticles on the flow properties of linear polymers: non-Einstein-like behavior, Macromolecules 38 (2005) 8000-8011.

[5] (a) A. Ruiz de Luzuriaga, A. Etxeberria, J. Rodríguez, J. A. Pomposo, Phase diagram and entropic interaction parameter of athermal all-polymer nanocomposites, Polym. Adv. Tech. (In press). DOI: 10.1002 / pat.1029. (b) For the sake of simplicity, in eq. 1 we have omitted a term $\left(\sim\right.$ constant $\left.\times \phi_{1}\right)$ arising from the (non-zero) reference free energy of the pure component 1 in the disordered molten state at $\phi_{1}^{f r}=0.494$ (maximum packaging of monodisperse spheres) as discussed in [5a] which has no effect in the resulting spinodal equation (second derivative of the free energy of mixing with respect to $\phi_{1}$ ).

[6] V. V. Ginzburg, Influence of nanoparticles on miscibility of polymer blends. A simple theory, Macromolecules 38 (2005) 2362-2367.

[7] T. Nishi, T. K. Kwei, Cloud point curves for poly(vinyl methyl-ether) and monodisperse polystyrene mixtures, Polymer 16 (1975) 285-290.

[8] G. Beaucage, R. S. Stein, T. Hashimoto, H. Hasegawa, Tacticity Effects on Polymer Blend Miscibility, Macromolecules 24 (1991) 3443-3448.

[9] I. C. Sanchez, R. H. Lacombe, Statistical thermodynamics of polymer solutions, Macromolecules 11 (1978) 1145-1156. 
[10] J. Dudowicz, K. F. Freed, Pressure dependence of polymer fluids: application of the lattice cluster theory, Macromolecules 28 (1995) 6625-6641.

[11] A. Mugica, J. A. Pomposo, M. Barral, M. Cortazar, Estimation of segmental interaction parameters in miscible blends, Proceedings of the $5^{\text {th }}$ GEP Meeting, Tarragona (1997).

[12] J. A. Pomposo, A. Mugica, J. Areizaga, M. Cortazar, Modeling of the phase behavior of binary and ternary blends involving copolymers of styrene, methyl methacrylate and cyclohexyl methacrylate, Acta Polymer. 49 (1998) 301-311. 